\title{
USE OF DECISION TREES IN COLOUR FEATURE SELECTION. APPLICATION TO OBJECT RECOGNITION IN OUTDOOR SCENES
}

\author{
J. Freixenet, X. Lladó, J. Martí, X. Cufí \\ Computer Vision and Robotics Group \\ Institute of Informatics and Applications. University of Girona. \\ Campus de Montilivi, 17071 Girona, Catalonia \\ \{jordif, llado, joanm, xcuf\}@eia.udg.es
}

\begin{abstract}
A new method for the automated selection of colour features is described. The algorithm consists of two stages of processing. In the first, a complete set of colour features is calculated for every object of interest in an image. In the second stage, each object is mapped into several ndimensional feature spaces in order to select the feature set with the smallest variables able to discriminate the remaining objects. The evaluation of the discriminate power for each concrete subset of features is performed by means of decision trees composed of linear discriminate functions.

This method can provide valuable help in outdoor scene analysis where no colour space has been demonstrated as being the most suitable. Experiment results recognizing objects in outdoor scenes are reported.
\end{abstract}

\section{INTRODUCTION}

Colour, being undoubtedly one of the most interesting characteristics of the natural world, can be computed in many different ways. Moreover, it is well known that chromatic characteristics of natural elements are not stable and highly dependant on colour brilliance, reflections from the object, illumination geometry, viewing geometry, and sensor parameters. Unfortunately, up to now no single solution has been found to sufficiently characterize objects belonging to natural scenes. So, a wide range of proposals has emerged all attempting to solve this problem [1]-[5].

When given an image representing an outdoor scene, the first difficulty in describing it is to choose the most appropriate colour space for object characterization. In this paper, we propose a new methodology to perform this operation which allows the user to choose the best colour features from among several colour spaces. The feature selection is performed for each object, starting with a series of samples obtained in a previously supervised learning task. This methodology enables a better and more specific object characterization, which improves the object recognition process, as has been demonstrated in experimental trials.

This paper is organized as follows. Section 2 presents our proposed colour feature selection algorithm. Some experimental results of natural scenes are presented to illustrate the performance of this approach. Finally, conclusions are explained in the last section.

\section{FEATURE SELECTION}

The proposed approach is based on a knowledge acquisition task intended as an interactive process to obtain sample data of the imaged objects. Initially, a teacher selects prominent examples of objects in training images by clicking on blobs of pixels. Afterwards, each object chosen is characterized by a large number of colour features deduced from different colour spaces. In order to obtain this initial characterization, it is possible to use as many features as wanted, adding new colour spaces if necessary. Table 1 indicates the 26 colour features used in our trials. These colour features represent some of the most used colour spaces in describing outdoor scenes. Once the data has been acquired, the algorithm selects the most interesting colour features for each object by comparing target from non-target instances. In this context "most interesting" means the features which separate the clusters of a feature space optimizing representation of the object samples. In this sense, we tackle two main challenges: a method to evaluate the discriminative power of a cluster in front of others, and a method of carrying out an intelligent combination of the whole set of colour features.

\subsection{Feature space evaluation}

The obtained data is calculated by means of functions which use the components $\mathrm{R}, \mathrm{G}$ and $\mathrm{B}$. This fact provokes 
Table 1. A set of 26 different colour features used for describing outdoor scenes.

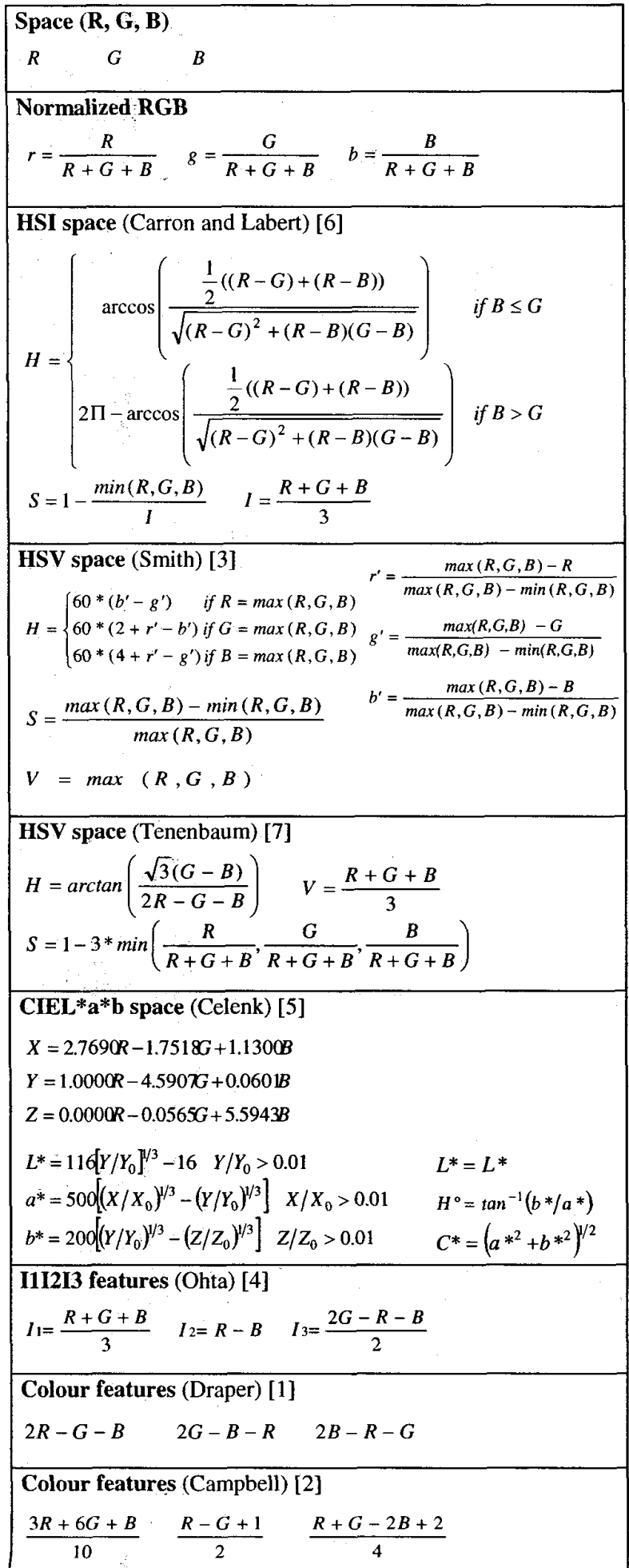

a clear functional relationship among these data, which means that some attributes add redundant information to the selection process. The proposed algorithm starts by eliminating features which are highly correlated. When two features are so closely repetitions, the system selects the one which has the lowest variance. Then, from a set of non-high-correlated features, a combinatorial process (described in section 2.2) selects which set has more discriminative power. This necessitates finding the space where the samples of the object make a compact cluster and are clearly separable from the rest. It is well known that there are two solutions to this problem: the linear and the non-linear approach. Following the paradigm of "divide and conquer" and using the linear approach, we have solved the problem by using a decision tree, which separates the samples recursively. Figure 1 shows the idea of the decision tree operating in a two-dimensional feature space.

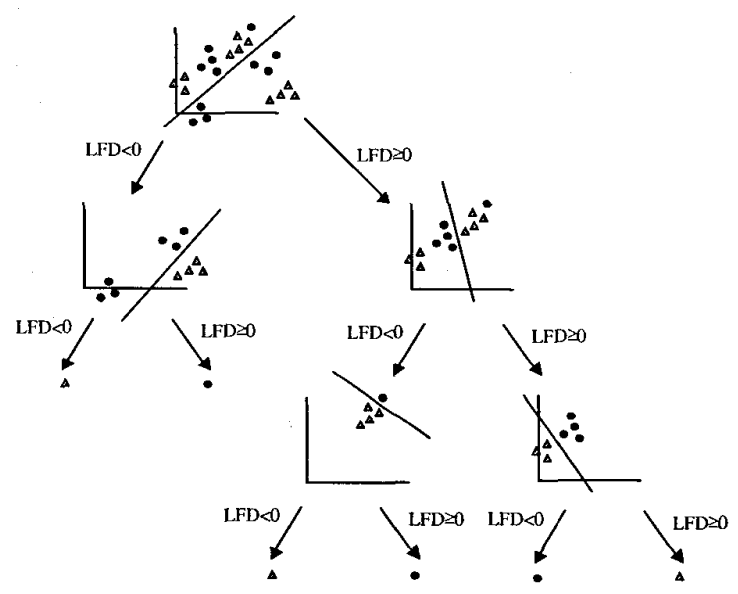

Figure 1. A decision tree in which linear functions recursively subdivide the set of samples between target and non-target.

The decision trees considered are binary trees with multivariate decision functions. Each node is a binary test represented by the Fisher LFD (Linear Discrimination Function).

$$
\left(\mu_{1}-\mu_{2}\right)^{t} \Sigma^{-1} x-\frac{1}{2}\left(\mu_{1}-\mu_{2}\right)^{t} \Sigma^{-1}\left(\mu_{1}+\mu_{2}\right)
$$

where:

$$
\begin{array}{ll}
\mu_{1} & \text { Means of all samples (Object). } \\
\mu_{2} & \text { Means of all samples (Rest of objects) } \\
\Sigma & \text { Matrix covariance. } \\
x & \text { Point to classify. }
\end{array}
$$

Each node in the tree attempts to separate target instances (mapped as •) in a set of known instances (the training set), from non-target instances (mapped as $\Delta$ ). The resulting two subsets of samples are subdivided again into 
two parts by using a new calculated Fisher LFD. This process is extended in a binary tree structure until an appropriated misclassification ratio is achieved. The tree construction process can be computably long. So, in order to solve this problem the tree is "pruned" by a threshold corresponding to a maximum depth.

\subsection{Selection algorithms}

In this section the process for choosing the best colour features for characterizing the objects is analyzed. We have proposed two ways to carry out this selection task: one in which the processing time is not a real constraint, and a second in which we try to achieve a real interaction with the system.

The best way to carry out the first alternative consists of performing all the combinations with the features (Exhaustive Search) and choosing the combination which most clearly defines the object of interest in front of the rest. Today this process still has a high computational cost and does not allow any real interactivity. In this sense, we propose to clip the combination process intelligently. This second proposal tries to shorten the whole combined exploration by pre-selecting a set of features using the Principal Component Analysis (PCA). This is one of the most frequently used algorithms to reduce data dimensionality in order to reduce the complexity of the timing task. PCA can be seen as a linear transformation which extracts a lower dimensional space which in turn reserves the major linear correlations in the data and discards the minor ones.

\section{EXPERIMENTAL RESULTS}

The performance of our proposal has been tested with a learning data set of 3000 points for each colour feature described in table 1 . These learning data are extracted from four different objects (Sky, leaves, road and ground) in an outdoor scene image. Table 2 shows the selected features of each object and the misclassification rate obtained with our proposal. Table 3 shows results obtained using some of the most typical colour spaces. The reliability of our proposal is proven through the data of the following tables.

Table 2. Misclassification rates obtained using our approach (number of features $=3$ and depth from the tree $=5$ ).

\begin{tabular}{|c|c|c|c|}
\hline object & Beatures & $\begin{array}{l}\text { Thee } \\
\text { lexel }\end{array}$ & $\begin{array}{l}\text { Visce } \\
\text { Rate }\end{array}$ \\
\hline Sky & G and $S$ (Smith) & 5 & 0 \\
\hline Leaves & S (Smith) & 2 & 0 \\
\hline Road & $\mathrm{I} 3, \mathrm{~S}$ (Smith) and $\mathrm{H}^{\circ}$ (Celenk) & 5 & 0.064 \\
\hline Ground & $\mathrm{H}$ (Smith) and $\mathrm{H}^{\circ}$ (Celenk) & 4 & 0 \\
\hline
\end{tabular}

Table 3. Misclassification rates obtained using three typical colour spaces (depth from the tree $=5$ ).

\begin{tabular}{|c|c|c|c|c|}
\hline & 4sky & Leaves & ERoad & Ground \\
\hline Teatures & $\begin{array}{c}\text { Rate/Tree } \\
\text { level }\end{array}$ & $\begin{array}{c}\text { Rate/Tree } \\
\text { level }\end{array}$ & $\begin{array}{c}\text { Rate/Tree } \\
\text { level }\end{array}$ & $\begin{array}{c}\text { Rate/Tree } \\
\text { level }\end{array}$ \\
\hline $\mathrm{R}, \mathrm{G}, \mathrm{B}$ & $0 / 3$ & $0.041 / 5$ & $0.076 / 5$ & $0 / 3$ \\
\hline H, S, V Smith & $0 / 4$ & $0 / 3$ & $0.078 / 5$ & $0.016 / 5$ \\
\hline H,S,V Tenenbaum & $0.002 / 5$ & $0 / 5$ & $0.101 / 5$ & $0.021 / 4$ \\
\hline
\end{tabular}

For example, figure 2 shows the distribution of the sample data by using different colour features. The set of features selected by our proposal gives a higher separability to the analyzed object (road) against the rest of the objects (see figure 2a). Furthermore, the cluster representing the road samples is more compact in figure $2 a$ than in figures $2 b, 2 c$, and $2 d$.

\section{CONCLUSIONS}

The proposed features selection approach provides a powerful tool in such situations where it is not clear which features are to be used in classification tasks, as happens in most outdoor scenes under variable lighting conditions. The ability to select the most appropriate features attenuates the lack of a consolidated colour space for characterizing outdoor scenes.

\section{REFERENCES}

[1] B.A. Draper, R.T. Collins, J. Brolio, A.R. Hanson, E.M. Riseman, "The Schema System", In International Journal of Computer Vision, Vol. 2. Pages 209-250. 1989.

[2] N.W. Campbell, W. Mackeown, B.T. Thomas, and T. Troscianko, "Interpretation Image Databases by Region Classification", In Pattern Recognition journal, Pages 555-563, April 1997.

[3] A.R. Smith, "Color gamut transform pairs", In Computer \& Graphics, 12(3):12-19, 1978.

[4] Y. Ohta, T. Kanade, and T. Sakai, "Color information for region segmentation", Computer Graphics and Image Processing, 13:222-241, 1980.

[5] M. Celenk, "A color clustering technique for image segmentation", Compute Vision, Graphics and Image Processing, 52:145-170, 1990.

[6] T. Carron and P. Lambert, "Color edge detector using jointly hue, saturation and intensity", In IEEE Int. Conf. on Image Processing, vol. 3, pages 977-981, 1994.

[7] J.N. Tenenbaum. "An Interactive facility for scene analysis research", Technical Note 87, Artificial Intelligence Center, Stanford Research Institute, 1974. 

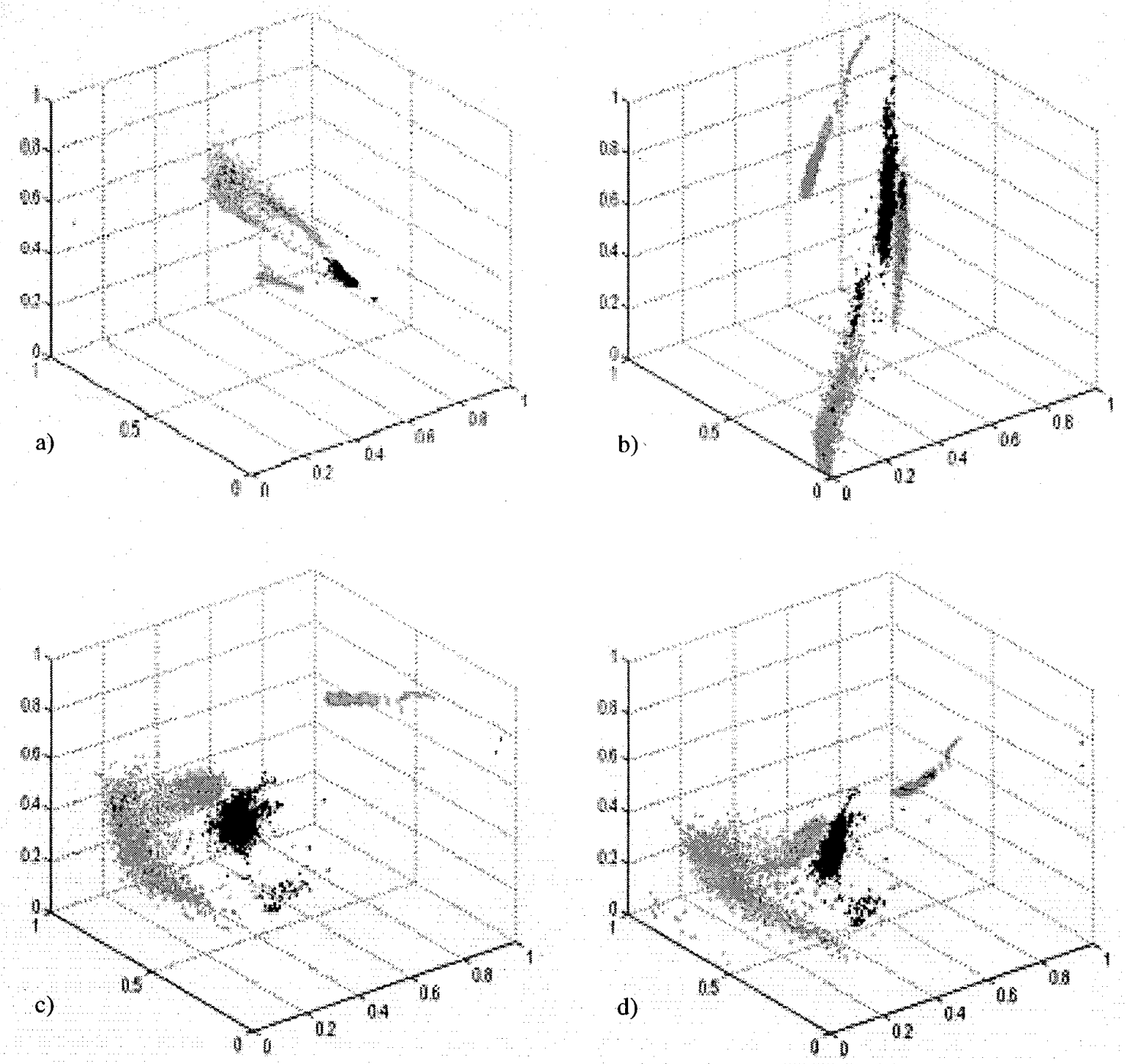

Figure 2. Road and non-road samples distribution in four different spaces. a) our proposal, b) RGB space, c) HSV Smith, d) HSV Tenenbaum. 